\title{
Velocity and Temperature of In-Flight Particles and Its Significance in Determining the Microstructure and Mechanical Properties of TBCs
}

\author{
Lei Zhang ${ }^{1} \cdot$ Tao He $^{2} \cdot$ Yu Bai $^{1} \cdot$ Fang-Li Yu ${ }^{3} \cdot$ Wei Fan $^{1} \cdot$ Yu-Shan $\mathrm{Ma}^{2} \cdot$ Zhan-Dong Chang ${ }^{2} \cdot$ Hai-Bo Liu $^{2} \cdot$ Ben-Qiang Li ${ }^{4}$
}

Received: 18 September 2018 / Revised: 21 November 2018 / Published online: 27 March 2019

(c) The Chinese Society for Metals (CSM) and Springer-Verlag GmbH Germany, part of Springer Nature 2019

\begin{abstract}
The correlation between particle in-flight parameter, defect content and mechanical property of yttria-stabilized zirconia coating was systematically studied in the present work. The melting state of in-flight particle during spraying was simulated using computational fluid dynamics. The results suggested that, with the increase of velocity and temperature of in-flight particles in the plasma jet, the particles changed from partially melted state to fully melted one. As a result, the total defect content of as-sprayed coating gradually decreased, while elastic modulus and microhardness increased correspondingly. However, the fracture toughness of as-sprayed coating reached a maximum value when the total defect content reached approximately $9.1 \%$.
\end{abstract}

Keywords Yttria-stabilized zirconia · Thermal barrier coating · Defect content · Computational fluid dynamics · Mechanical property

\section{Introduction}

The demand of improving effectiveness of energy conversion has driven significant efforts for improving the service temperature of gas turbine engines. One of the effective ways is applying a ceramic thermal barrier coating (TBC) on the surface of metallic turbine blade to reduce its service temperature $[1,2]$. The development of a TBC system focuses on the choice of new materials, structural design and property evaluation [3-8]. As a commonly used TBC material, yttria-stabilized zirconia (YSZ) cannot be replaced in a short term due to its low thermal conductivity and high thermal

Available online at http://link.springer.com/journal/40195

Yu Bai

byxjtu@mail.xjtu.edu.cn

$\checkmark$ Yu-Shan Ma

mys@wzyb.com.cn

1 State Key Laboratory for Mechanical Behavior of Materials, Xi' an Jiaotong University, Xi' an 710049, China

2 Wuzhong Instrument Co. Ltd, Wu Zhong 751100, China

3 School of Materials Engineering, Xi' an Aeronautical University, Xi' an 710077, China

4 Department of Mechanical Engineering, University of Michigan, Dearborn, MI 48128, USA expansion coefficient, which is similar to that of the metallic substrate.

A typical TBC system consists of a thin oxidation-resistant metallic bond coat which is sandwiched between the substrate and thermally grown oxides (TGOs) with a layer of YSZ top coat. The latter is usually deposited by electron beam-physical vapor deposition (EB-PVD) or air plasma spraying (APS). Compared to the EB-PVD, APS can fabricate TBC with a higher efficiency and a lower thermal conductivity. However, the APS process is very sensitive to the parameter variations. The previous study showed that there were over 35 main macroscopic parameters which significantly affected the microstructure of as-sprayed coatings [9]. Additionally, other elements, such as power injection, geometrical fluctuation and electrode erosion, cannot be effectively controlled. Thus, it is an important challenge for engineers and scientists to tailor the microstructure of as-sprayed coatings.

Three separate, but interrelated processes during plasma spraying include plasma generation and plasma-particle interactions, heating and acceleration of in-flight particles, splat stacking and coating formation. These processes can be employed to control the behavior of moving particles and to tailor the microstructure of as-sprayed coating. However, it is difficult to measure the thermal and fluid fields of plasma jet or to experimentally observe the heating and 
acceleration of in-flight particles. The development of computational fluid dynamics (CFD) has enabled the study of velocity and temperature of in-flight particles during spraying [10-12]. From the technological and scientific perspectives, the velocity and temperature of in-flight particle, prior to their impact onto the substrate, are two major parameters that influence the microstructure of coatings, since they significantly affect the flattening behaviors of in-flight particles [13, 14]. Additionally, due to continuous flattening and quick solidification of melted or partially melted particles, the as-sprayed coating consists of lamellar structures and many types of defects, such as cracks, unmelted particles and pores $[15,16]$.

Although many studies have analyzed the microstructure and properties of plasma-sprayed coatings [17-19], a detailed correlation among particle in-flight parameter, defect content and mechanical property of YSZ coatings is still scarce. Based on the above background, in the present work, a physical model was built in order to describe compressible reacting flow and its interaction with particles. The procedure contained the mass transfer, particle melting and solid-liquid phase transition. Meanwhile, the relationship among particle in-flight parameter, defect content and mechanical property of YSZ coatings was established.

\section{Experimental}

\subsection{Materials}

The substrate, stainless steel plate with dimensions of $10 \mathrm{~mm} \times 5 \mathrm{~mm} \times 5 \mathrm{~mm}$, was ultrasonically cleaned in acetone and grit-blasted using alumina for the purpose of increasing its adherence capability with the bond coat. A commercially CoNiCrAlY powder (AMDRY 995M, Sulzer Metco Inc., USA) with a nominal composition of Y-0.4, Al-8, Cr-21, $\mathrm{Ni}-32$ and Co-balance (wt \%) was used to deposit the bond coat. One sintered and spray-dried $\mathrm{ZrO}_{2}-8 \mathrm{wt} \% \mathrm{Y}_{2} \mathrm{O}_{3}$ powder with a size of $10-55 \mu \mathrm{m}$ was used to deposit the top coat. The morphology and inner structure of YSZ powder are shown in Fig. 1.

\subsection{Plasma Spraying Process}

The ceramic top coat with a thickness of $200 \mu \mathrm{m}$ was deposited by a high-efficiency supersonic atmospheric plasma spraying (SAPS) system and a Metco 9M air plasma spraying (APS) system, respectively. The spray parameters are listed in Table 1. In terms of SAPS and APS, three different operating conditions, respectively, are described as high $V-T$ (487-513 m/s, 3544-3579 K) (SAPS), medium $V-T(441-468 \mathrm{~m} / \mathrm{s}, 3307-3455 \mathrm{~K})$ (SAPS) and low $V-T$ $(211-221 \mathrm{~m} / \mathrm{s}, 3150-3291 \mathrm{~K})$ (APS) as listed in Table 2.

\subsection{Interaction Between Plasma Jet and In-Flight Particles}

The computational model concerning plasma jet as well as its interaction with the in-flight particles was established using CFD-ACE software (ESI Group, France) as shown in Fig. 2. The particles in the flow were treated as the separate Lagrangian entities. The governing equations for the Reynolds averaged flow parameters were dealt with finite volume strategy. The momentum and continuity equations were coupled by pressure-implicit with splitting of operators (PISO) algorithm. In the computational model of single particle, the diameter of YSZ particle was set as $40 \mu \mathrm{m}$ according to the median diameter obtained from a laser particle size analyzer (Rise-2008, Jinan Rise Science and Technology Co., Ltd., Jinan, China). The density, thermal conductivity, melting point and specific heat capacity of YSZ material were $5.89 \mathrm{~g} / \mathrm{cm}^{3}, 2.0 \mathrm{~W} /(\mathrm{m} \mathrm{K}), 2950 \mathrm{~K}$ and $580 \mathrm{~J} /(\mathrm{kg} \mathrm{K})$, respectively. The inlet boundary situation for the momentum and thermal transfer was based on the flow field features within the nozzle exit. The gases contained argon and hydrogen, and the inlet pressure was $1000 \mathrm{~Pa}$.
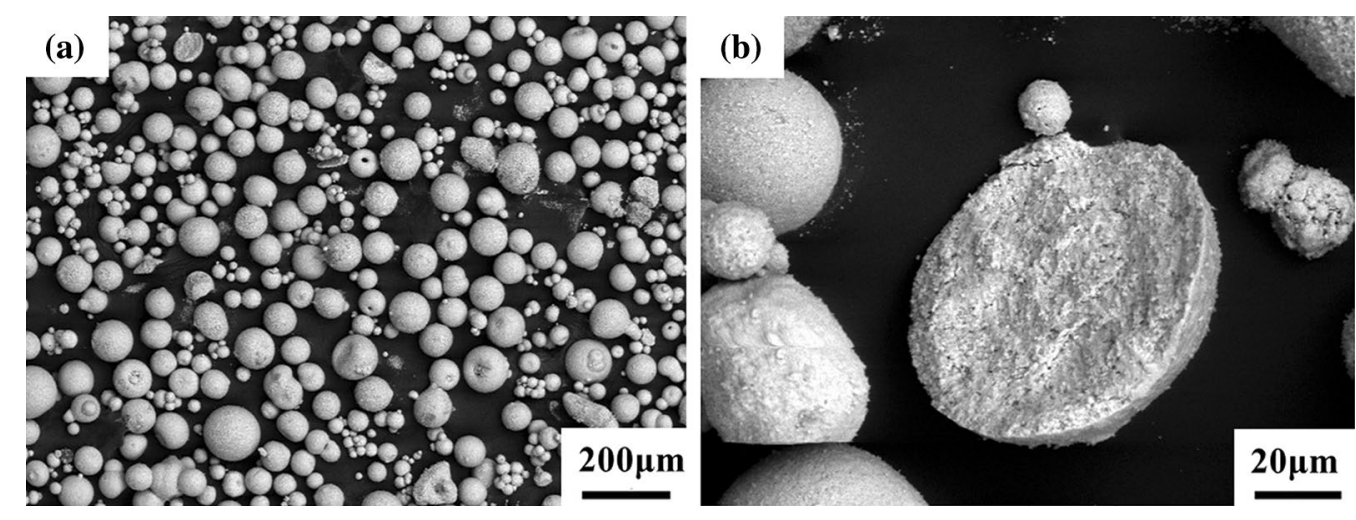

Fig. 1 Morphologies of original YSZ feedstock powders: a whole view of powders; $\mathbf{b}$ a detailed view of crushed single powder 
Table 1 Spray parameters of YSZ coatings

\begin{tabular}{llllllll}
\hline No. & Spraying method & Current (A) & $\begin{array}{l}\text { Primary gas } \\
\text { Ar (slpm) }\end{array}$ & $\begin{array}{l}\text { Secondary } \\
\text { gas } \mathrm{H}_{2}(\mathrm{slpm})\end{array}$ & $U(\mathrm{~V})$ & $\begin{array}{l}\text { Stand- } \\
\text { off distance } \\
D(\mathrm{~mm})\end{array}$ & $\begin{array}{l}\text { Feed- } \\
\text { ing rate } \\
\left(\mathrm{g} \mathrm{min}^{-1}\right)\end{array}$ \\
\hline S1 & SAPS & 504 & 65.0 & 19.5 & 118 & 90 & 35 \\
S2 & SAPS & 472 & 75.0 & 21.0 & 124 & 90 & 35 \\
S3 & SAPS & 487 & 70.0 & 21.0 & 123 & 90 & 35 \\
S4 & SAPS & 356 & 60.3 & 15.0 & 125 & 90 & 35 \\
S5 & SAPS & 366 & 65.0 & 15.4 & 131 & 90 & 35 \\
S6 & SAPS & 312 & 65.2 & 16.7 & 133 & 90 & 35 \\
S7 & APS & 605 & 44.7 & 25.0 & 75.6 & 70 & 40 \\
S8 & APS & 654 & 42.3 & 27.0 & 74.5 & 70 & 40 \\
S9 & APS & 651 & 47.0 & 12.5 & 72.6 & 70 & 40 \\
S10 & APS & 648 & 51.7 & 15.0 & 73.8 & 70 & 40 \\
\hline
\end{tabular}

Table 2 Velocity and temperature of in-flight YSZ particles in the plasma jet

\begin{tabular}{lllrl}
\hline Samples & Velocity $(\mathrm{m} / \mathrm{s})$ & $\begin{array}{l}\text { Temperature } \\
(\mathrm{K})\end{array}$ & $\begin{array}{l}\text { Total defect } \\
\text { content }(\%)\end{array}$ & Description \\
\hline $\mathrm{C} 1$ & $490 \pm 4$ & $3579 \pm 3$ & $6.9 \pm 0.4$ & High $V-T$ \\
$\mathrm{C} 2$ & $513 \pm 3$ & $3544 \pm 2$ & $9.1 \pm 0.6$ & High $V-T$ \\
$\mathrm{C} 3$ & $487 \pm 2$ & $3552 \pm 2$ & $9.4 \pm 0.9$ & High $V-T$ \\
$\mathrm{C} 4$ & $453 \pm 4$ & $3335 \pm 6$ & $10.2 \pm 0.5$ & Medium $V-T$ \\
$\mathrm{C} 5$ & $468 \pm 3$ & $3455 \pm 4$ & $10.9 \pm 0.8$ & Medium $V-T$ \\
$\mathrm{C} 6$ & $441 \pm 2$ & $3307 \pm 3$ & $11.5 \pm 0.4$ & Medium $V-T$ \\
$\mathrm{C} 7$ & $212 \pm 2$ & $3291 \pm 5$ & $13.4 \pm 0.6$ & Low $V-T$ \\
$\mathrm{C} 8$ & $221 \pm 2$ & $3258 \pm 5$ & $15.4 \pm 0.9$ & Low $V-T$ \\
C9 & $213 \pm 2$ & $3173 \pm 4$ & $18.3 \pm 0.6$ & Low $V-T$ \\
C10 & $211 \pm 2$ & $3150 \pm 5$ & $20.5 \pm 0.7$ & Low $V-T$ \\
\hline & & & &
\end{tabular}

The outlet velocity and pressure were set as $0 \mathrm{~m} / \mathrm{s}$ and normal atmospheric pressure, respectively. The morphology of particle was supposed to be spherical with a stable size during the change of solid-liquid phase. The evaporation of particle was neglected during the melting process. The plasma governing equations and corresponding heating and accelerating model can be found elsewhere [12].

\subsection{Specimen Characterization}

The velocity and temperature of in-flight particle obtained by Spray Watch 2i (Oseir, Finland) online system are presented in Table 2. The porosity, unmelted particle content and crack content were quantitatively measured by ImagePro Plus software (Media Cybernetics, America). After the conversion of grayscale images to binary ones, the statistical porosity, unmelted particle content as well as crack content were obtained from ten cross-sectional scanning electronic microscopy (SEM) images with $800 \times$ magnification.

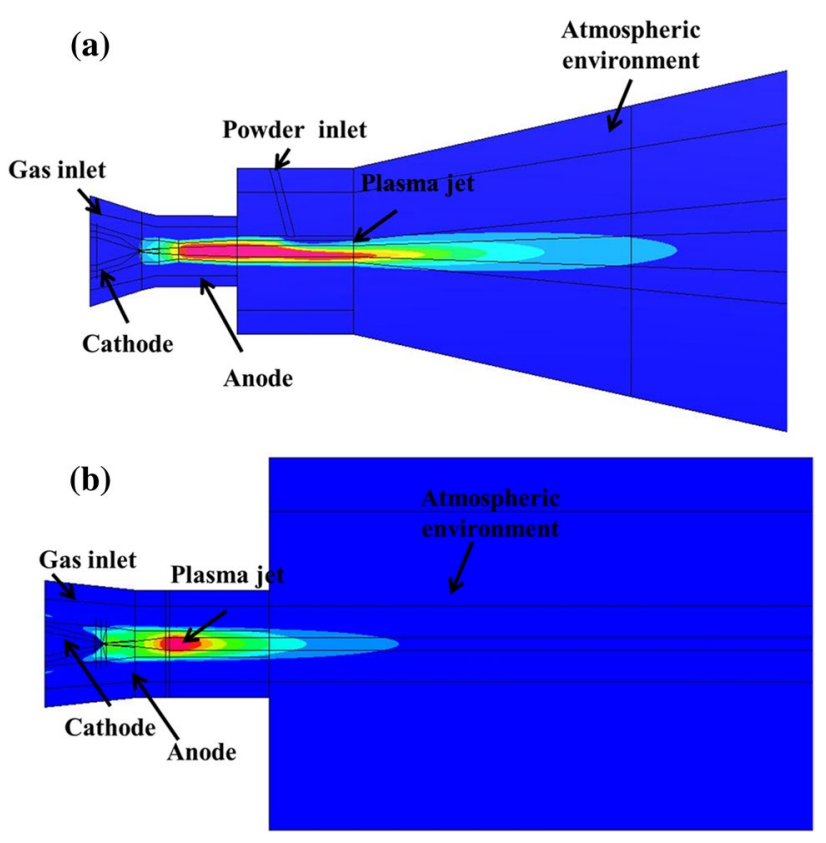

Fig. 2 Computational model of plasma spraying: a SAPS; b APS

\subsection{Microhardness and Weibull Analysis}

The microhardness of as-sprayed coating was measured by a microhardness tester (MicroMet 3 microhardness tester, Buehler Ltd., USA). The polished cross section of the coating was measured 30 times at different areas with a load of $2.94 \mathrm{~N}$ and a holding time of $10 \mathrm{~s}$ while maintaining a distance between consecutive indentations at least five times greater than the diagonal length of indentation. The microhardness data scatter was treated via Weibull statistical analysis. The two-parameter Weibull distribution function was utilized and given as [20-22]: 
$F(x)=1-\exp \left[-\left(\frac{x}{x_{0}}\right)\right]^{m}$,

where $F(x)$ is the cumulative density function of probability; $x$ is the microhardness data; $x_{0}$ is the scale factor, which gives $63.2 \%$ of the cumulative density; and $m$ is the Weibull modulus. The Weibull modulus reflects the data scatter in the distribution. The Weibull plot is the most common and easiest technique for obtaining the Weibull modulus. A Weibull plot can be drawn by rearranging Eq. (1) and taking natural logarithms twice. Thus, $x_{0}$ and $m$ can be determined by fitting the following equation:

$\ln \left\{\ln \left[\frac{1}{1-F(x)}\right]\right\}=m\left[\ln (x)-\ln \left(x_{0}\right)\right]$.

The mean value of $F(x)$ is obtained by arranging the data in ascending order and letting [22]:

$F(x)=\frac{i-0.3}{n+0.4}$

where $i$ is the $i$ th element in an ascending data set and $n$ is the total number of data points.

The elastic modulus of as-sprayed coating was measured as follows [23, 24]:

$\frac{b^{\prime}}{a^{\prime}}=\frac{b}{a}-\alpha \frac{H_{\mathrm{v}}}{E}$

where $a^{\prime}$ and $b^{\prime}$ are the diagonal dimensions after the Knoop indentation's elastic recovery, $a$ and $b$ are the diagonal dimensions before the Knoop indentation's elastic recovery and $H_{\mathrm{v}}$ is the Knoop hardness.

The fracture toughness of as-sprayed coating was measured by the following equation [25]:

$K_{\mathrm{IC}}=k_{\text {total }}\left[\frac{P}{a \sqrt{L}}\right]$,

where $k_{\text {total }}$ is double the value of $k_{\mathrm{p}}$ ( $k_{\mathrm{p}}$ is an empirical constant and equals to 0.0319 in Palmqvist pattern); $P$ is the indentation load (with a value of $2.94 \mathrm{~N}$ in this study); $L$ is the surface cracks' overall length, containing all cracks surrounding the indentation; and $a$ is the indentation halfdiagonal on average. Meanwhile, $a$ and $L$ are calculated as follows:

$a=\frac{2 a_{1}+2 a_{2}}{4}$

$L=\sum_{n} l_{y n}$

\section{Results and Discussion}

\subsection{Analysis of Plasma Jet and Melting State of In-Flight Particles}

The velocity and temperature of plasma jet without any particles are shown in Fig. 3. As seen from Fig. 3, the highest velocity and temperature of plasma jet were $1936 \mathrm{~m} / \mathrm{s}$ and 15,260 K in SAPS. Meanwhile, the highest velocity and temperature of plasma jet were $658 \mathrm{~m} / \mathrm{s}$ and $17,820 \mathrm{~K}$ in APS, respectively. Figure 4 shows vertical sectional contours of velocity and temperature of plasma jet at the nozzle exit. The velocity and temperature were significantly influenced by the carrier gas. Their distributions presented a symmetric "double-peaked hump" shape, as shown in Fig. 4. The highest velocity and temperature areas moved downward from the nozzle center. The key advantage in SAPS system was associated with an inner powder injection port, which was located in the nozzle and is perpendicular to the plasma jet. This design guaranteed that the injected powder can acquire a higher velocity and temperature. In addition, although the highest temperature of the plasma jet under low $V-T$ condition was slightly higher than other conditions (Fig. 3), the temperature at the nozzle exit was still the lowest.

Figure 5 shows the cross-sectional temperature contour of single particle at the end of in-flight time. As seen from Fig. 5, the temperature in the periphery was higher than that in the center of the particle. Meanwhile, owing to the impact of carrier gas, the lowest temperature was towards the tail of gas flow. Because the evaporation was neglected, the temperature of particle was increasing steadily. The lowest temperature of a particle was $3150 \mathrm{~K}$ under high $V-T$ condition (Fig. 5a), indicating that the YSZ particle was completely melted. However, they maintained a constant value below $2950 \mathrm{~K}$ under medium $V-T$ or low $V-T$ condition, suggesting that the YSZ powder was partially melted prior to its impact on the substrate.

\subsection{Microstructure of As-Sprayed Coating}

A plasma-sprayed coating was constituted by a sequence of thermal and mechanical interactions of in-flight particles with the substrate. These events determined the microstructure of as-sprayed coating. Figure 6 depicts a typical morphology of splat deposited onto a mirrorpolished substrate under different spray parameters. As seen from Fig. 6, many cracks formed on the surface of splats due to the relief of quenching stress. The surface roughness $(\mathrm{Ra})$ increased from 0.6 to $1.2 \mu \mathrm{m}$ when the spray parameters varied from high $V-T$ condition to low 


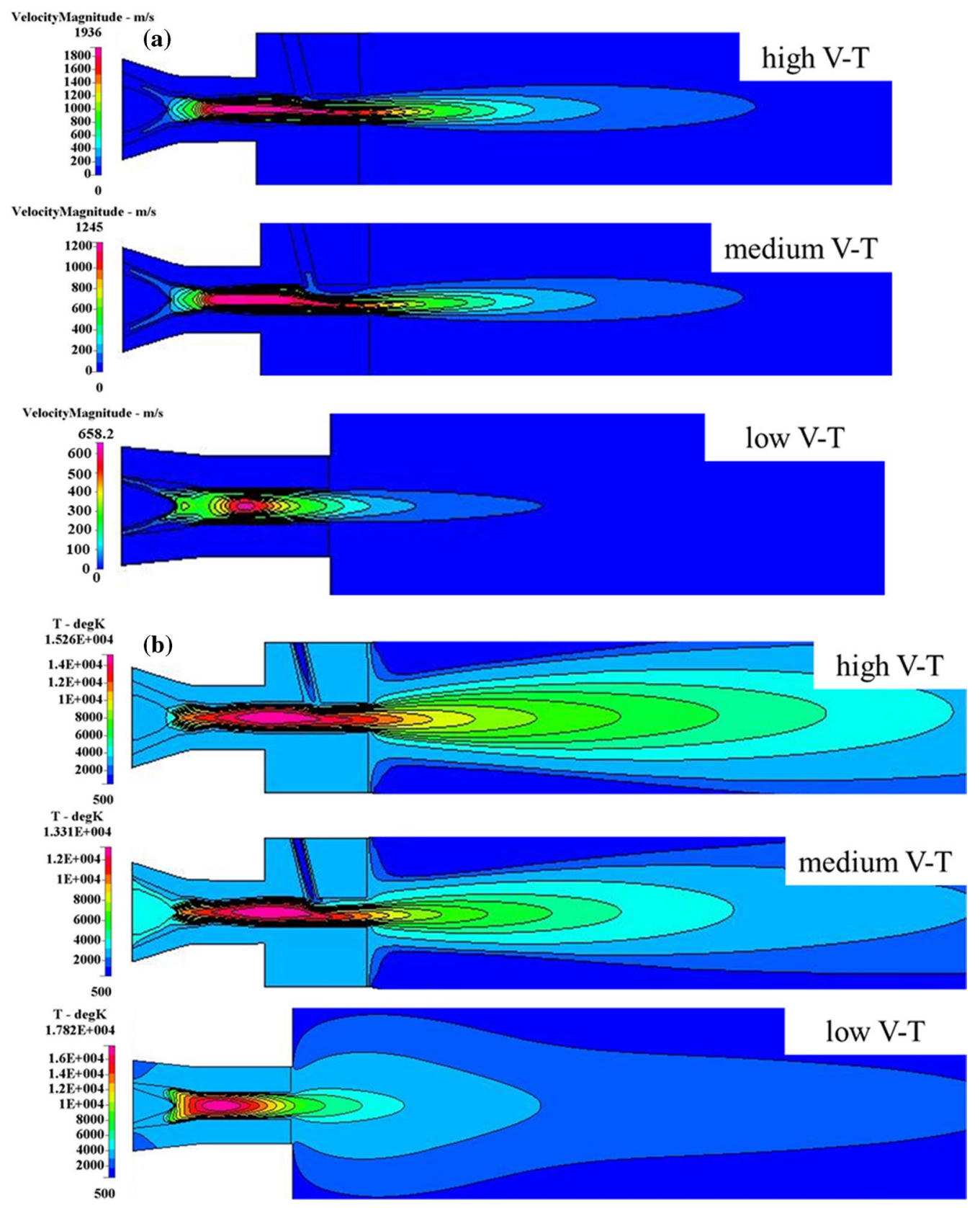

Fig. 3 Velocity and temperature distribution of plasma jet with various spray parameters: a velocity distribution; $\mathbf{b}$ temperature distribution

$V-T$ one. This can be explained by the radial spread of molten particle. For the low $V-T$ condition, the so-called multilayer solidification occurred when the moving fronts of liquid waves were arrested by a previously solidified wave as shown in Fig. 6. In addition, as shown in Fig. 6a, $\mathrm{b}$, the molten particle significantly splashed on the substrate surface under the medium or high $V-T$ condition. On the contrary, a near disk-shaped splat formed under low $V-T$ condition, indicating an insufficient spreading.
The insufficient spreading may cause incomplete filling of splats and resultant defects, such as pores in the coating.

Figure 7 gives the cross-sectional morphologies of assprayed coatings. It is clear from Fig. 7 that the various defects increased with the decrease of velocity and temperature of in-flight particles. Only a few fine pores, unmelted particles and cracks were observed under high $V-T$ condition (Fig. $7 \mathrm{a}-\mathrm{c}$ ), while a large number of inter-lamellar coarse pores, unmelted particles and cracks were found under low $V-T$ condition (Fig. $7 \mathrm{~g}-\mathrm{i}$ ). The melting state of in-flight 
Velocity Magnitude - $\mathrm{m} / \mathrm{s}$

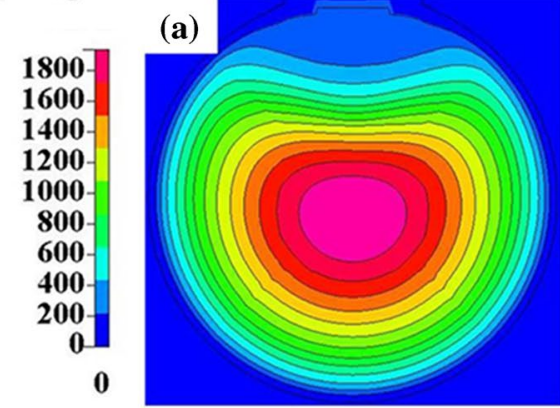

High V-T

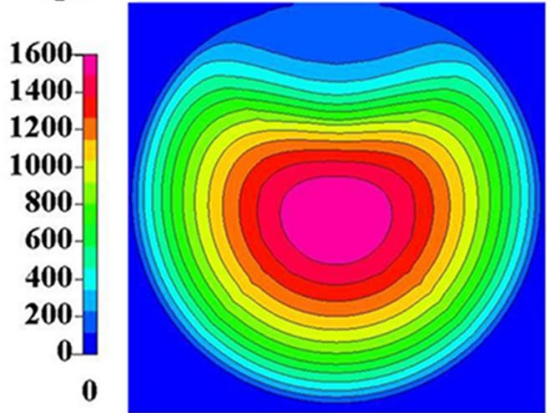

Velocity Magnitude - m/s Medium V-T

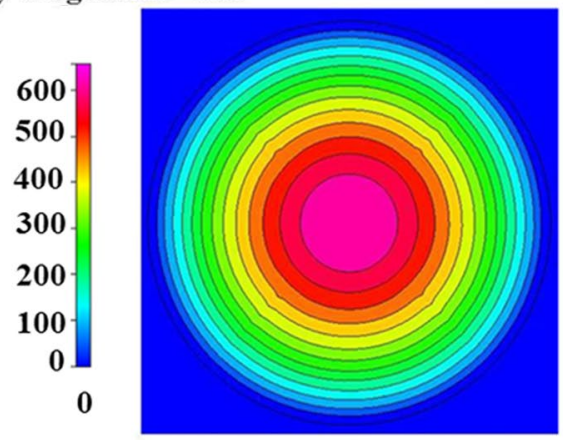

Low V-T

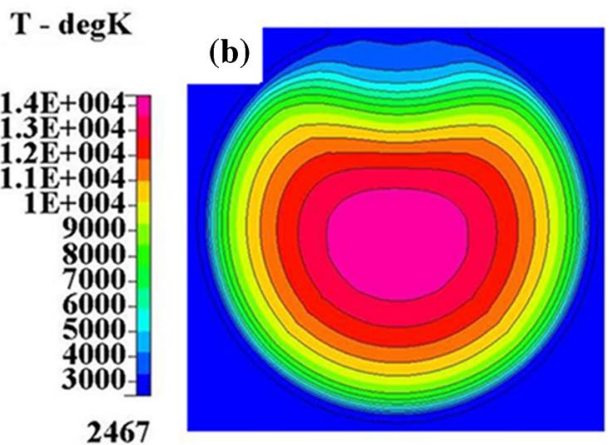

High V-T

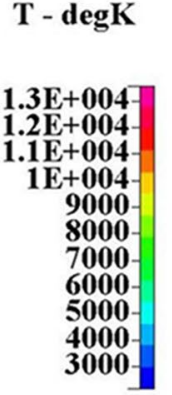

2180

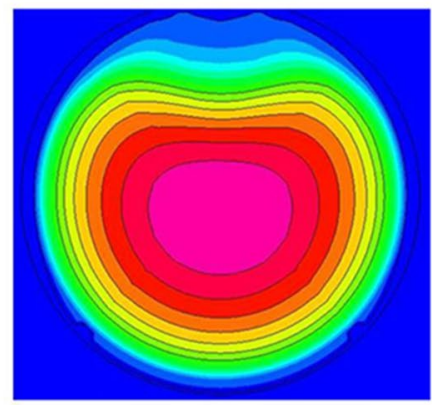

Medium V-T

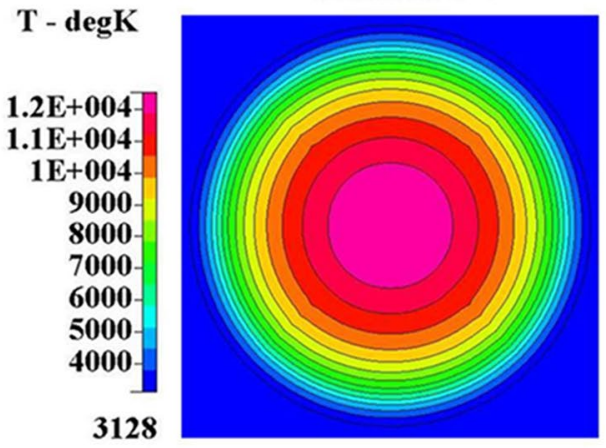

Low V-T

Fig. 4 Cross-sectional contour of plasma jet at nozzle exit with different spray parameters: a velocity distribution; b temperature distribution
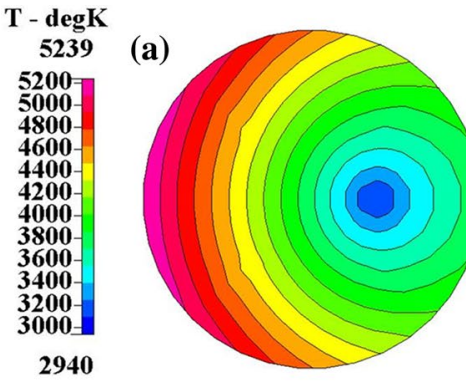

high V-T
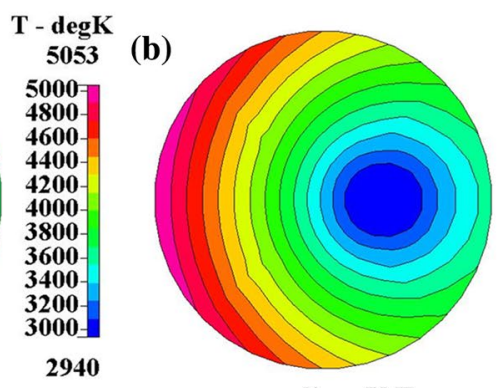

medium V-T
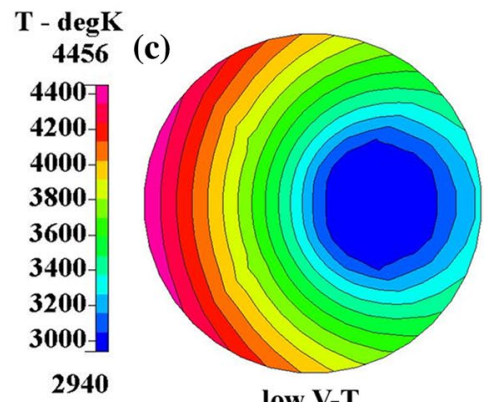

Fig. 5 Temperature distribution of single particle at the end of in-flight time: a high $V-T$ condition; b medium $V-T$ condition; $\mathbf{c}$ low $V-T$ condition 

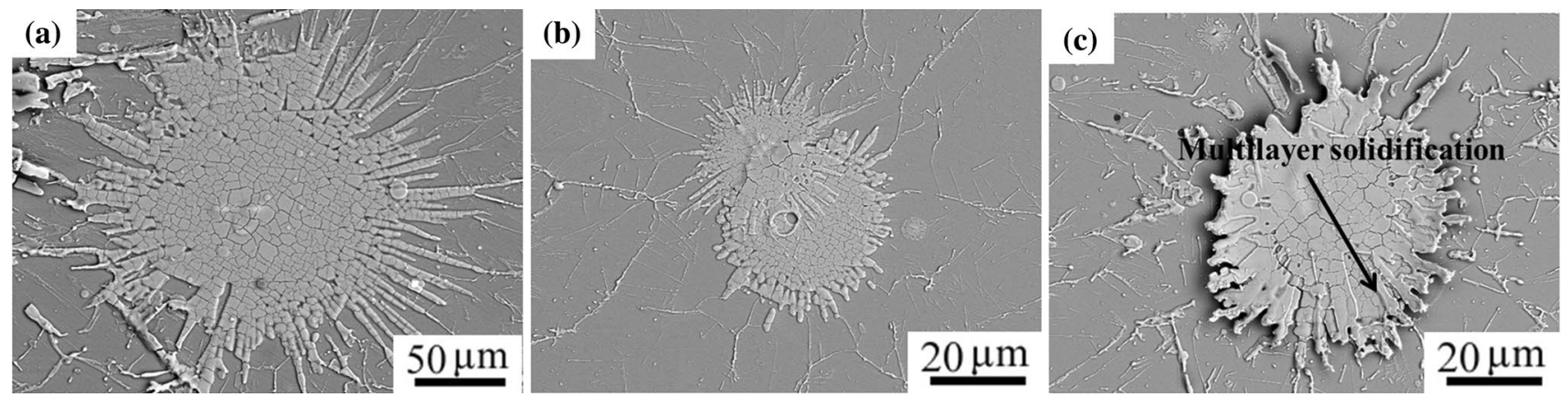

Fig. 6 Morphologies of splats deposited on mirror-polished substrates: a high $V-T$ condition; b medium $V-T$ condition; $\mathbf{c}$ low $V-T$ condition
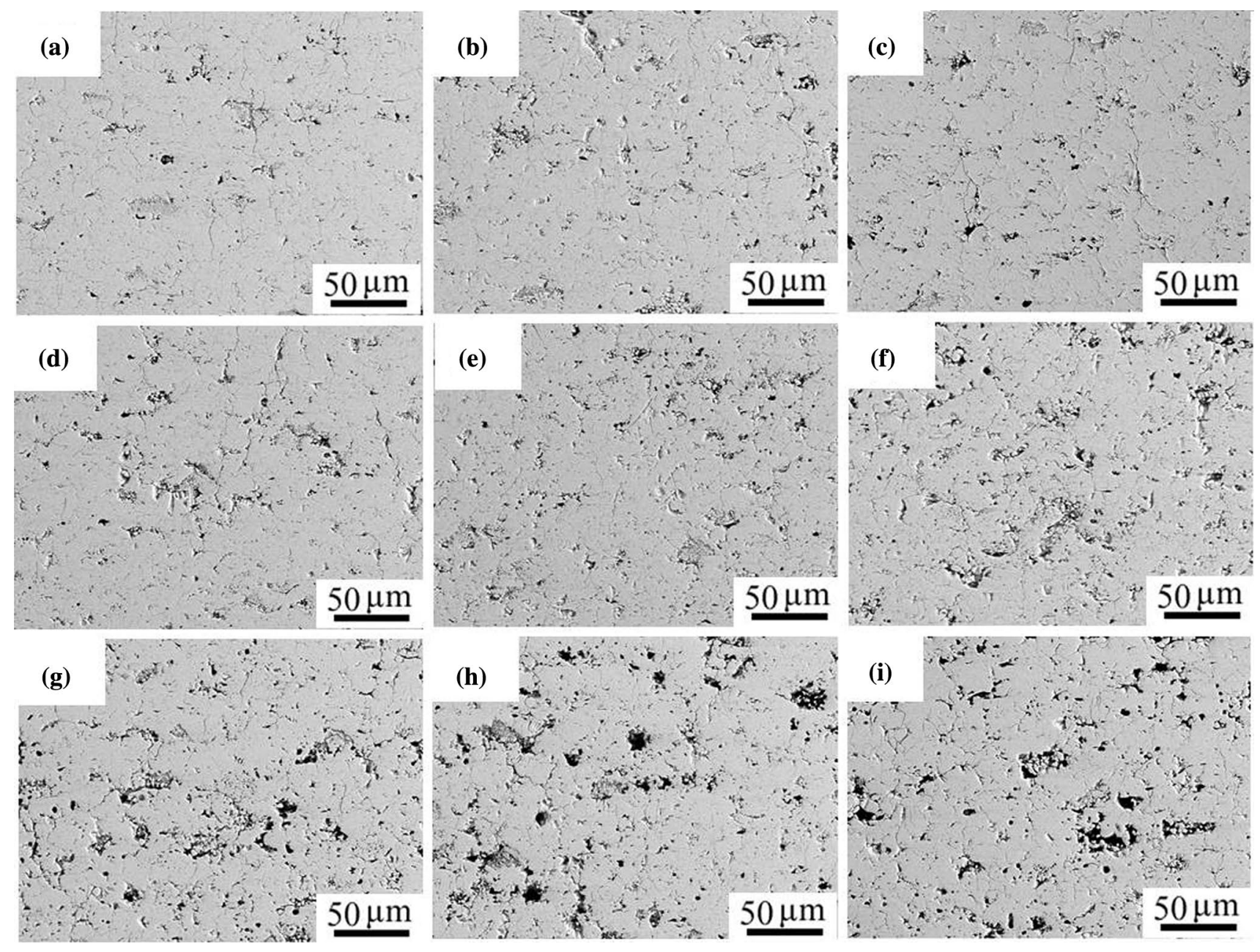

Fig. 7 Cross-sectional microstructure of as-sprayed coatings: a C1; b C2; c C3; d C4; e C5; f C6; g C7; h C8; i C9

particle was strongly dependent on the momentum and energy transfer between the injected particle and plasma jet. With respect to a single injected particle, the surface temperature is higher than the center one. Nevertheless, the high surface temperature could not guarantee the complete melting of particle. Thus, a lot of unmelted particles were kept at the central part of the original feedstock powder and accompanied by the crystalline area originated from the melted part of starting powder. The latter performed as a binder and kept the coating integrity. There were two distinct pore morphologies in the as-sprayed coating: one was large-sized pores (approximately $3-10 \mu \mathrm{m}$ ), which was mainly caused 
by the incomplete contact of splats (as shown in Fig. 7g-i); the other was fine pores (approximately $0.01-0.1 \mu \mathrm{m}$ ), which was originated from the entrapped gas during coating formation [26, 27]. Other defects, such as cracks, could form due to the relaxation of thermal or quenching stresses during spraying.

Figure 8 gives the statistical total defect content, porosity, crack content and unmelted particle content as a function of velocity and temperature of in-flight particles. As shown in Fig. 8, when the velocity decreased from $490 \pm 4$ to $210 \pm 2 \mathrm{~m} / \mathrm{s}$ and the temperature reduced from $3306 \pm 3$ to $2877 \pm 5 \mathrm{~K}$, the porosity, crack content and unmelted particle content increased by $233 \%, 123 \%$ and $286 \%$, respectively. The above results suggested that the
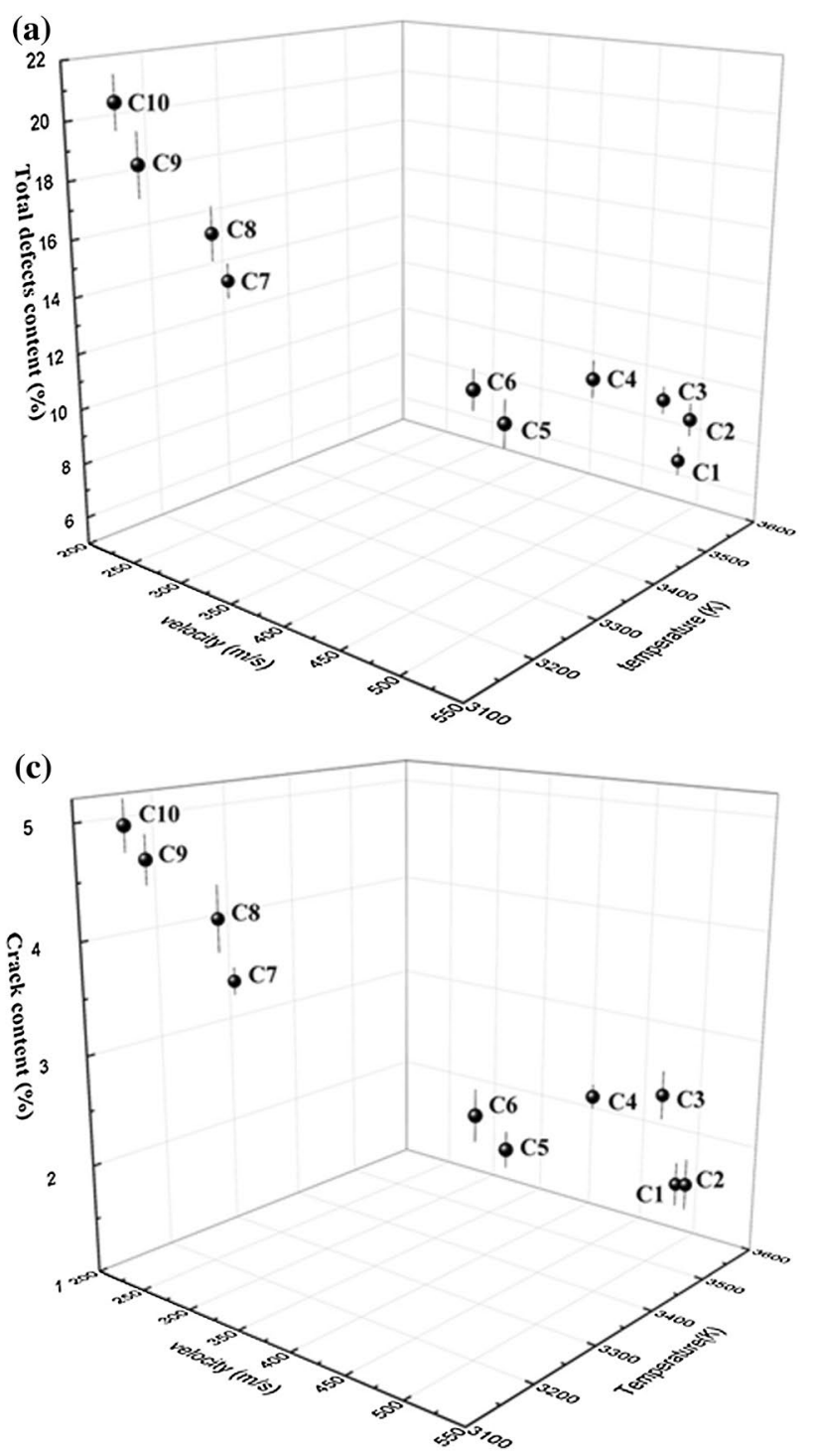

in-flight particles under low $V-T$ condition possessed the lowest melting state, which resulted in the highest unmelted particle contents in $\mathrm{C} 10$ coating. In addition, the porosity, crack content as well as unmelted particle content decreased with the increase of the velocity and temperature of in-flight particles. Particle temperature was a predominant factor in controlling the defect content, especially when the velocity variations were limited during spraying. The in-flight particles with lower temperatures may result in an increase in large pores or unmelted particles in the coating. Meanwhile, the in-flight particles with lower velocity may lead to an imperfect contact of splats and an increase in smaller-sized pores when they
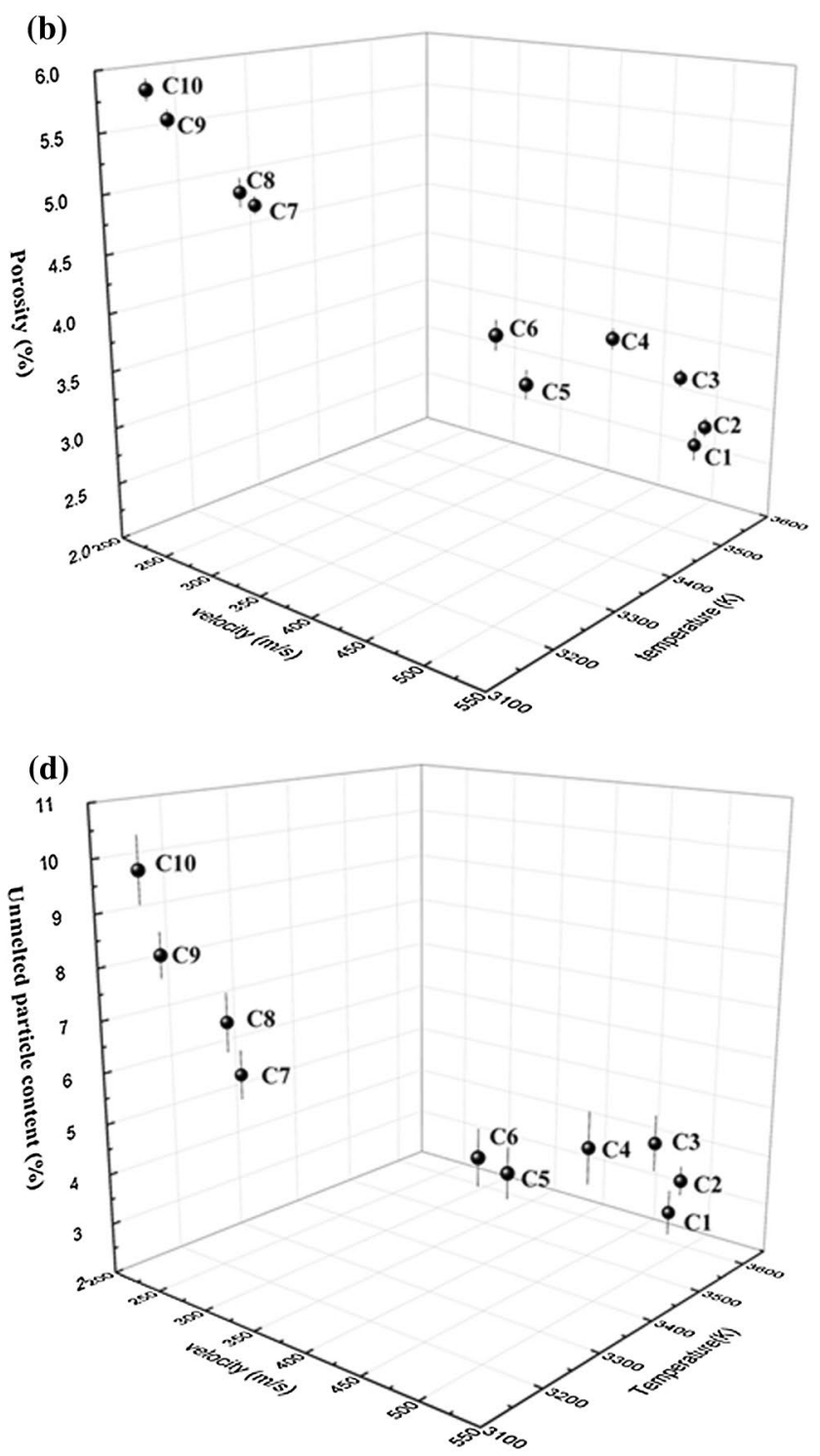

Fig. 8 Statistical results of a total defect content, $\mathbf{b}$ porosity, $\mathbf{c}$ crack content and $\mathbf{d}$ unmelted particle content as a function of velocity and temperature of in-flight particles 
successively impacted onto the substrate or previously deposited splats.

\subsection{Microhardness and Weibull Plots}

Figure 9a presents the Weibull plots' probability function of microhardness of as-sprayed coatings. There was obvious scatter in Vickers hardness owing to a non-uniform microstructure of as-sprayed coating. As shown in Fig. 9b, the Weibull modulus $M$ ranged from 12.39 to 5.58. The value of $M$ increased with increasing velocity and temperature of in-flight particles, indicating a higher microstructural homogeneity. The Vickers hardness of as-sprayed coatings fell in the scope of $884 \pm 84.2 \mathrm{H}_{\mathrm{v} 0.3} /$ $\mathrm{kg} \mathrm{mm} \mathrm{m}^{-2}$ to $726 \pm 101.6 \mathrm{H}_{\mathrm{v} 0.3} / \mathrm{kg} \mathrm{mm}^{-2}$. Additionally, the microhardness of as-sprayed coating increased with increasing velocity and temperature of in-flight particles. The low microhardness under low $V-T$ condition can be ascribed to the high porosity or unmelted particle content.

\subsection{Elastic Modulus and Fracture Toughness}

The elastic modulus, determined by the Knoop indentation technique, is on the basis of the elastic recovery measurement of a Knoop indentation. Elastic deformation is mainly due to the propagation of pores and cracks [28, 29]. This differs markedly from that of the bulk material according to the following empirical relationship:

$E=E_{0} \exp \left(-b p+c p^{2}\right)$

where $p$ is the volume fraction porosity, $E_{0}$ is the dense material's modulus, and $b$ and $c$ are values that consider the pore morphology [30]. It can be directly inferred from Eq. 8 that the elastic modulus increased with the decrease of total defect content. Figure 10 depicts the elastic modulus or fracture toughness as a function of total defect content. The elastic modulus decreased from $177.8 \pm 6.9$ to $112.1 \pm 9.5 \mathrm{GPa}$ when the total defect content increased from 6.9 to $20.5 \%$. The reason can be explained by the fact that the elastic recovery was inhibited due to the higher defect content [24, 31]. Figure 11 gives the fracture toughness of as-sprayed coatings as a function of unmelted particle content. As seen

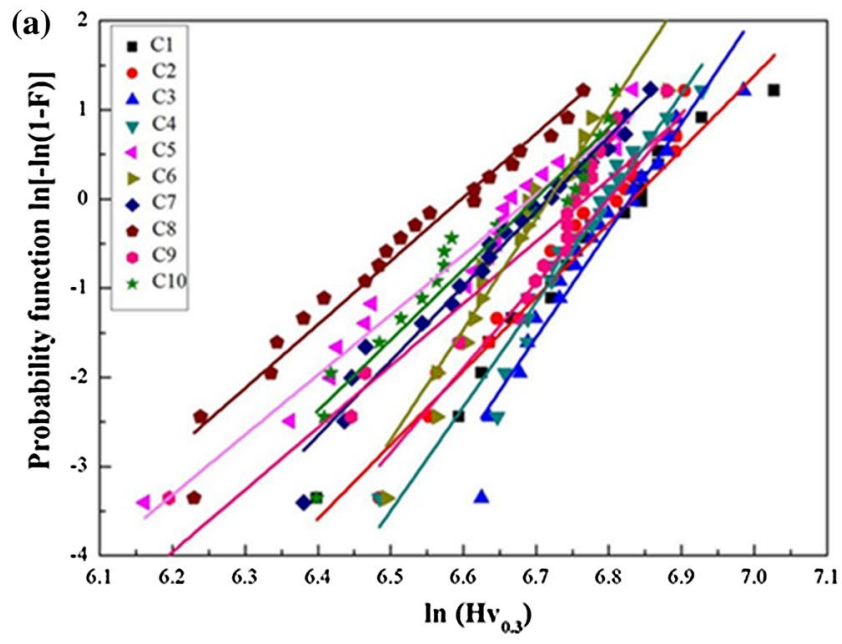

(b)

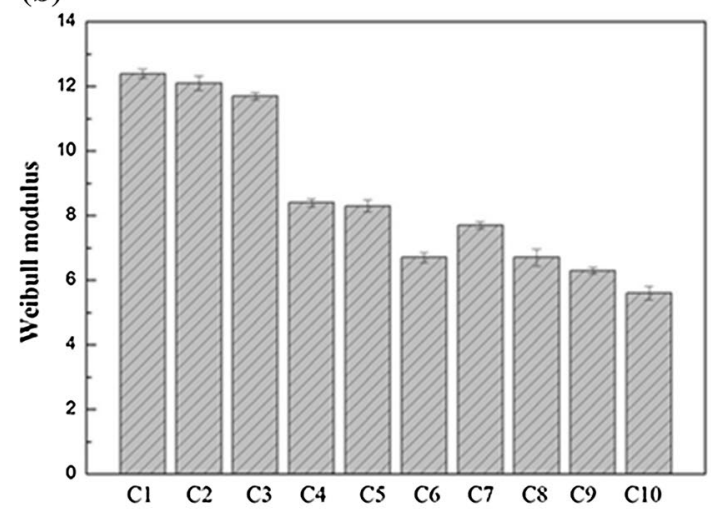

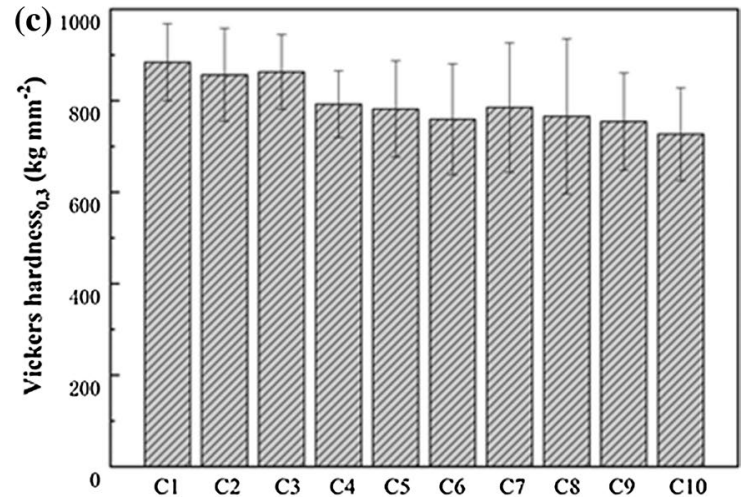

Fig. 9 a Weibull distribution; b Weibull modulus; c Vickers hardness of as-sprayed coatings 


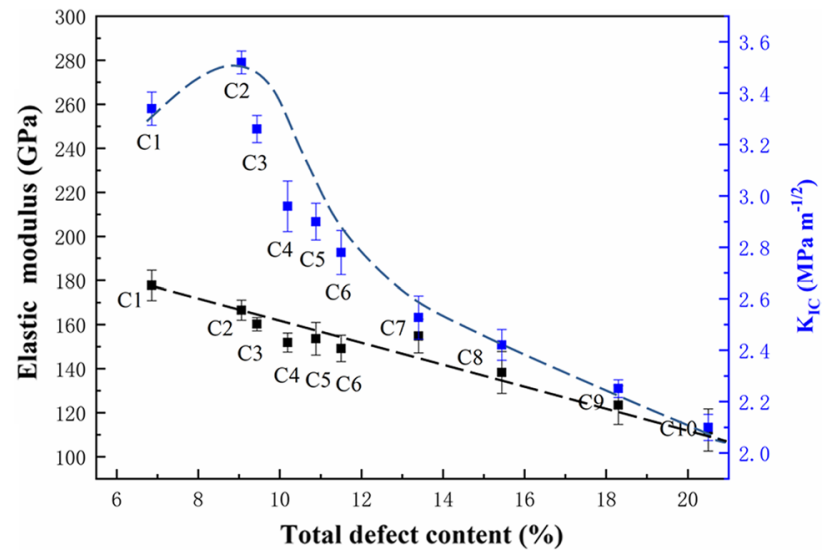

Fig. 10 Elastic modulus and fracture toughness of as-sprayed coatings as a function of total defect content

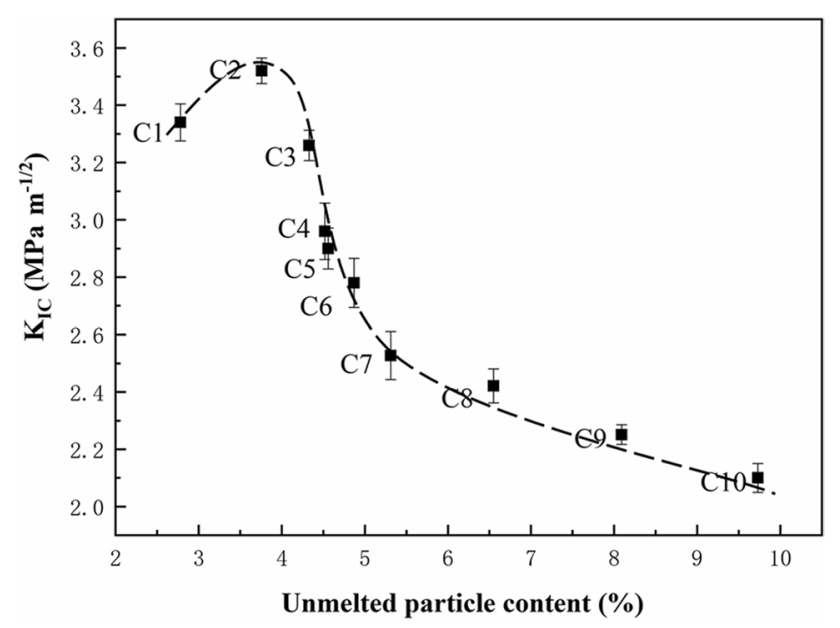

Fig. 11 Fracture toughness of as-sprayed coatings as a function of unmelted particle content

from Figs. 10 and 11, the fracture toughness first increased, then decreased and maximized when the total defect content reached approximately $9.1 \%$.

According to Eq. 5, both microhardness and total surface crack length $L$ have a significant influence on the fracture toughness of as-sprayed coating. The unmelted particles with a medium content can effectively inhibit the propagation of cracks. However, the excessive unmelted particle may result in the decrease in toughness because some cracks can initiate and propagate along the interface between the crystalline area and unmelted particles. Indentation-induced cracks generated and propagated along the diagonal direction, as shown in Fig. 12. The indentation presented a typical continuous and deflected morphology of cracks at the corner of indentation when the cracks met various defects. The indentation shows a remarkable increase in length of crack and a significant reduction in fracture toughness from high $V-T$ condition to low $V-T$ one. It can be also found that the fracture toughness of as-sprayed coating was in the range from $3.56 \pm 0.11$ to $2.10 \pm 0.20 \mathrm{MPa} \mathrm{m}^{-1 / 2}$. However, it was clear that many cracks stopped propagating after their tip met some unmelted particles (Fig. 12b). The unmelted particles that embedded in the coating performed as "crack arresters" and thus improved the toughness of as-sprayed coating. Nevertheless, if the lamellar structures were disrupted by immoderate unmelted particles, lots of cracks can initiate and propagate along the crystalline area/unmelted particles interface due to the weak inter-lamellar bonding, leading to the reduction in fracture toughness (Fig. 12h). In addition, for $\mathrm{C} 1$ and $\mathrm{C} 2$ coating, although the microhardness decreased with increasing the defects, the stress at the crack tip can be relieved by the unmelted particles with medium content. From $\mathrm{C} 3$ to $\mathrm{C} 10$ coating, with decreasing the velocity and temperature of in-flight particles, the melting state got worse and the increased total defects decreased the fracture toughness of as-sprayed coating. Several microcracks were also observed near the tips of coarse cracks (Fig. 12a), which helped to release the residual stress and toughen the coating. During plasma spraying, due to the combination of spray parameters, such as arc current, argon or hydrogen flow rate and spray distance, the velocity as well as temperature of plasma jet decreased from high $V-T$ condition to low $V-T$ one. The melting state of in-flight particle was highly dependent on its velocity and temperature. The successive impingement of overlapped individual splats constituted the as-sprayed coating and inevitably formed several types of defects including pores, cracks and unmelted particles. These defects decided the mechanical properties of coating. Therefore, the microstructure and mechanical properties of coating can be tailored by controlling the defect contents through modulating the velocity and temperature of in-flight particles.

\section{Conclusions}

In this study, the correlation among particle in-flight parameters, defect content and mechanical properties of YSZ coatings was systematically studied. Some conclusions can be drawn as follows:

(1) With the increase of velocity and temperature, the melting state of in-flight particle was significantly enhanced, resulted in the decrease of total defect content including porosity, unmelted particle content and crack content of as-sprayed coatings.

(2) Microhardness and elastic modulus of as-sprayed coating gradually decreased with the increase of the total defect content. Fracture toughness of as-sprayed coating first increased, then decreased and reached a maxi- 

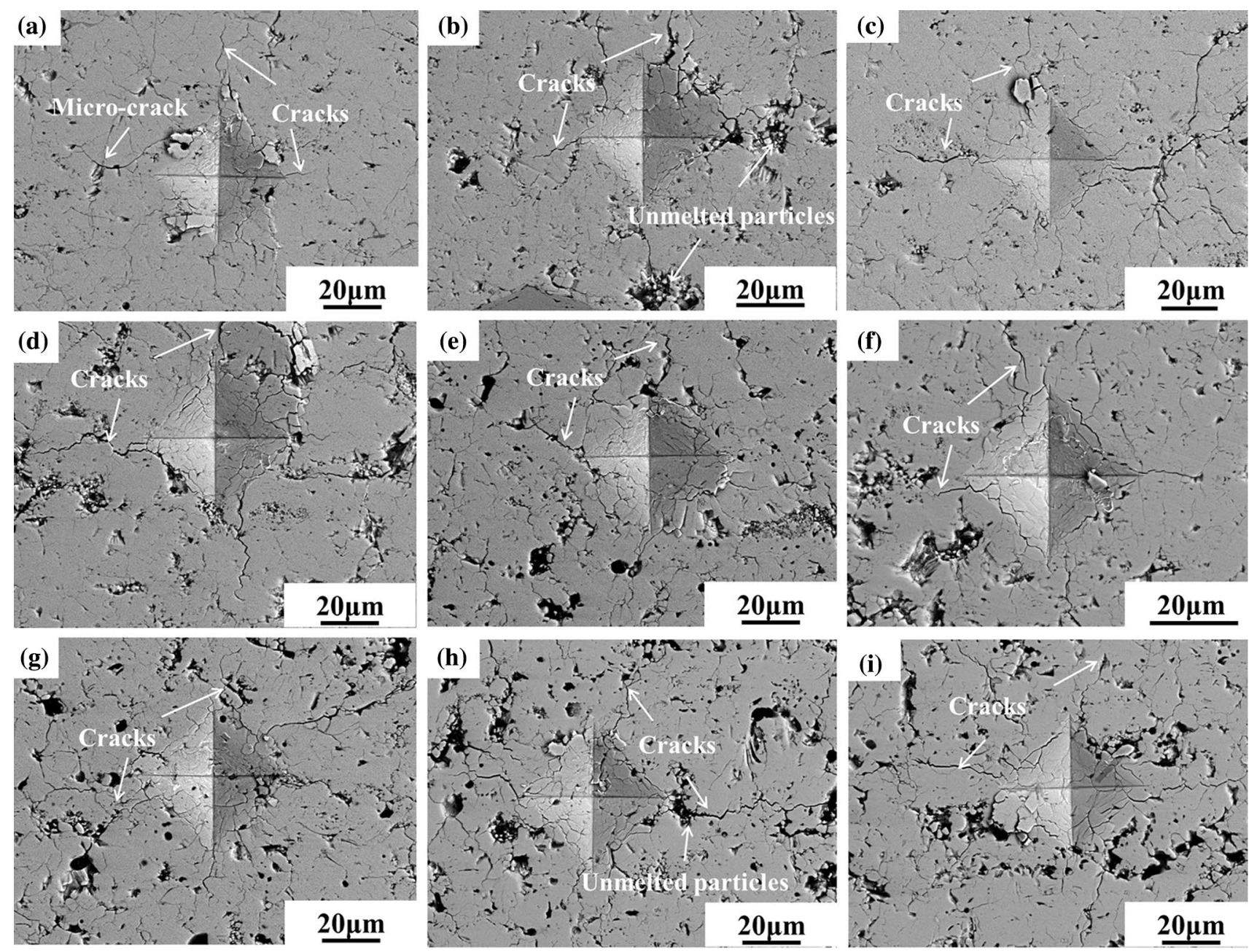

Fig. 12 Cross-sectional morphology of Vickers indentations and crack propagation of as-sprayed coatings: a C1; b C2; c C3; d C4; e C5; f C6; $\mathbf{g}$ C7; h C8; i C9

mum value when the total defect content was approximately $9.1 \%$ due to the inhibition of unmelted particles on the propagation of cracks.

(3) The microstructure and mechanical properties of assprayed YSZ coating can be tailored by controlling the defect contents through modulating the velocity and temperature of in-flight particles.

Acknowledgements This work was supported by the Collaborative Innovation Center of Advanced Control Valve Project (Grant No. WZYB-XTCX-001). The authors would like to thank Mr. Zijun Ren at Instrument Analysis Center of Xi' an Jiaotong University for the assistance with SEM analysis.

\section{References}

[1] J.C. Williams, E.A. Starke, Acta Mater. 5775, 51 (2003)

[2] N.P. Padture, M. Gell, E.H. Jordan, Science 280, 296 (2002)
[3] R. Vassen, X.Q. Cao, F. Tietz, D. Basu, D. Stöver, J. Am. Ceram. Soc. 2023, 83 (2010)

[4] J. Zhang, X.Y. Guo, Y.G. Jung, L. Li, J. Knapp, Surf. Coat. Technol. 18, 323 (2017)

[5] W.B. Li, L.L. Liu, Y.F. Yang, S.L. Zhu, F.H. Wang, Acta Metall. Sin. (Engl. Lett.) (2018). https://doi.org/10.1007/s40195-0180842-1(0123456789

[6] C.S. Che, J.L. Lu, G. Kong, Q.Y. Xu, Acta Metall. Sin. (Engl. Lett.) 138, 22 (2009)

[7] S.M.A. Shibli, K.S. Chinchu, M.A. Sha, Acta Metall. Sin. (Engl. Lett.) (2018). https://doi.org/10.1007/s40195-018-0823-4

[8] S.H. Wang, C.S. Liu, F.J. Shan, Acta Metall. Sin. (Engl. Lett.) 161, 22 (2009)

[9] A. Vardelle, M. Vardelle, P. Fauchais, Plasma Chem. Plasma P. 255, 2 (1982)

[10] D.Q. Zhang, L.L. Zheng, X.Y. Hu, H. Zhang, Int. J. Heat Mass Transf. 508, 98 (2016)

[11] H.B. Xiong, L.L. Zheng, S. Sampath, R.L. Williamson, J.R. Fincke, Int. J. Heat Mass Transf. 5189, 47 (2004)

[12] P. Wei, Z.Y. Wei, G.X. Zhao, J. Du, Y. Bai, Comput. Mater. Sci. 8, $103(2015)$

[13] Y. Bai, Z.H. Han, H.Q. Li, C. Xu, Y.L. Xu, Z. Wang, C.H. Ding, J.F. Yang, Appl. Surf. Sci. 7210, 257 (2011) 
[14] P. Fauchais, J. Phys. D Appl. Phys. 86, 37 (2004)

[15] T. Nakamura, G. Qian, C.C. Berndt, J. Am. Ceram. Soc. 578, 83 (2000)

[16] S. Deshpande, A. Kulkarni, S. Sampath, H. Herman, Surf. Coat. Technol. 6, 187 (2004)

[17] A. Vaidya, V. Srinivasan, T. Streibl, M. Friis, W. Chi, S. Sampath, Mater. Sci. Eng. A. 239, 497 (2008)

[18] Z. Wang, A. Kulkarni, S. Deshpande, T. Nakamura, H. Herman, Acta Mater. 5319, 51 (2003)

[19] T. Yang, J.P. Longtin, S. Sampath, J. Am. Ceram. Soc. 710, 92 (2009)

[20] C.K. Lin, C.C. Berndt, J. Mater. Sci. 111, 30 (1995)

[21] A. Dey, A.K. Mukhopadhyay, S. Gangadharan, Ceram. Int. 2295, 35 (2009)

[22] H. Zhou, F. Li, B. He, Surf. Coat. Technol. 7360, 201 (2007)

[23] S.W. Kweh, K.A. Khor, P. Cheang, Biomaterials 1223, 21 (2000)
[24] K.A. Khor, Y.W. Gu, C.H. Quek, P. Cheang, Surf. Coat. Technol. 195, 168 (2003)

[25] N.H. Faisal, R. Ahmed, A.K. Prathuru, S. Spence, M. Hossain, J.A. Steel, Eng. Fract. Mech. 189, 128 (2014)

[26] R.S. Lima, A. Kucuk, C.C. Berndt, Mater. Sci. Eng. A 224, 327 (2002)

[27] X.C. Zhang, B.S. Xu, Y.X. Wu, F.Z. Xuan, S.T. Tu, Appl. Surf. Sci. 3879, 254 (2008)

[28] H.J. Kim, Y.G. Kweon, Thin Solid Films 201, 342 (1999)

[29] Z.Y. Yu, Z.Q. Tan, G.L. Fan, R.B. Lin, D.B. Xiong, Q. Guo, Y.S. Su, Z.Q. Li, D. Zhang, Acta Metall. Sin. (Engl. Lett.) 1121, 31 (2018)

[30] J. Wang, J. Mater. Sci. 801, 19 (1984)

[31] Y. Wang, Y. Bai, T. Yuan, H.Y. Chen, Y.X. Kang, W.J. Shi, X.L. Song, B.Q. Li, Surf. Coat. Technol. 95, 319 (2017) 